\title{
Automatic Control System of the smart transfer device based on S120
}

\section{and PLC}

\author{
Xiuyuan WU ${ }^{1, a}$, Weichuang QI ${ }^{1, b}$, Zhiyi ZHANG ${ }^{1, c}$, Yongjun LIU ${ }^{2, d}$, \\ Xiangyang $\mathrm{WU}^{1, \mathrm{e}}$
}

1. Department of Technology Engineering, CSR Qingdao Sifang Co., Ltd., Shandong, Qingdao, 266111;

2. Welding Institute, Southwest Jiaotong University, Sichuan, Chengdu, 610031

a wuxiuyuan@cqsf.com, bqwc5137@163.com, c yefan_0954@163.com, ${ }^{\mathrm{d}} \mathrm{lyj}$ jllxIII@163.com, esfxiangyangw@163.com

Keywords: transfer device, Control system, S120, Programmable Logic Controller

Abstract: The transfer device is widely used in the industrial production line, and the different production lines have different requirements for the equipment control system. In the paper, a smart transfer device control system based on the S120 and PLC was developed for a production line with two different products, and it can transfer each product in different mode. The system uses the master-slave control mode, which PC is upper computer and the S7-300 PLC is lower computer. The data exchange between PLC and S120 is achieved by Profibus, and the data between PLC and $\mathrm{PC}$ is exchanged by Ethernet. The system is equipped with Human Machine Interface, which can meet the requirements of high precision control of the transfer device, and solve the complex problem of multi-parameter control and multi-function control. The result of field debugging verified that the system is stable, easy to operate, and has strong practicability.

\section{Introduction}

With the development of industrial technology, the transfer device is widely used in the production line, which helps to transfer products or semi-finished products. At present, the transfer machine has two types: with rail and without rail[1]. The transfer device with rail has limited moving range, but the system precision is high, and it can transport the heavy workpieces. The transfer device without rail is more flexible, but the positioning precision is limited to the existing technology, and it is difficulty to transport heavy workpieces. The existing equipment control system is mainly based on PLC or PC motion control card, in which the PLC system is relatively simple and the PC system is mainly used in the tunnel type stacking machine [2,3]. The transfer device is mainly used for point-to-point transport, how to develop its control system is an important issue for the logistics of the factory in the condition of multi-station, multi-products, high-precision and multi-function[4,5]. In this paper, the production line includes assembly station, welding station, repair welding station and grinding station, etc. It has welding robots, automatic grinding machines and other equipments. The workpiece mainly includes two types and their semi-finished products, which the structure of them is complicated. To realize the automation of this production line, it is important to develop a control system of the smart transfer device that can coordinate each station and transfer the product effectively. Therefore, the paper develops an smart transfer device control system based on S120 and PLC. 


\section{The design of the control system for the smart transfer device}

The transfer device is an important component of the production line, which is connecting all the parts of the production line. Its basic functions includes taking and discharge of workpiece, which is mainly depending on the walking mechanism of $\mathrm{X}$ direction, the fork telescopic mechanism of $\mathrm{Y}$ direction and the lifting mechanism of $\mathrm{Z}$ direction . Therefore, the system must control the action of these mechanisms. The walking mechanism and lifting mechanism are responsible for the precise positioning of the workpiece, and the fork telescopic mechanism is responsible for taking the workpiece. Therefore, the requirements of the control accuracy are higher, for example, the control accuracy of walking mechanism is within the range of $1 \mathrm{~mm}$. Designing the control system of the smart transfer device, it is necessary to solve the problem of zero position and speed control, that is, it can determine the three-dimensional coordinates of the device at any time[6].Because of the fixed position and clear division of labor, automatic identification of the workpiece type can be solved by the layout of the station. In addition, the system also requires that when a failure occurs, the production can't be effects more. At the same time the system should facilitate on-site operation and meet the production line management.

According to the above requirements, the control system uses Sinamics S120 to achieve the movement control. The Sinamics S120 can be used as an independent system, and it can also be used to form a more complex control system with PLC. S120 is a drive control system which integrates V/F, vector control and servo control. It can not only control three-phase asynchronous motor, but also can control the synchronous motor, torque motor, etc. Its powerful positioning function will realize the absolute and relative position of feed motion. The control unit adopts CU320, the DP PROFIBUS bus control mode, which makes the structure compact, easy to install and debug. According to the control accuracy requirements of the system positioning, the walking mechanism and fork telescopic mechanism use respectively single servo motor with absolute value encoder. The driving motor of the lifting mechanism adopts two three-phase asynchronous motor with an incremental encoder, and the speed of the two motor is synchronous. The identification of workpiece type is realized by means of process layout. In order to cope with the possible failure of the intelligent transfer device, the system is set up to four operation models: maintain, manual, semi-automatic and automatic. In order to facilitate the operation and management, the system designed a movable touch screen and developed a Human-Machine Interface. At the same time, it designed a fixed type PC machine to facilitate management. Among them, PC and PLC use Ethernet communication to achieve data exchange, touch screen and PLC use PROFIBUS DP to achieve data exchange, PLC and S120 use PROFIBUS DP to achieve data exchange. In addition, the system also deals with the related signals, such as the signal in place, the detection signal, the starting signal and so on. The control system structure of the intelligent transfer device is shown in Fig.1.

The system separates the power module and the motor module. The power module is connected to the DC bus of $560 \mathrm{~V}$ and the motor module is connected to the DC bus. The system uses the modular structure, embodies the advantage of flexibility and structure in terms of additional functions and debugging.

The control function of the intelligent transfer device includes the movement control, position control and speed control of the walking mechanism, the fork telescopic mechanism and the lifting mechanism. It is easy to realize the movement control of the walking mechanism, the fork telescopic mechanism and the lifting mechanism, and the speed control[7] is achieved by motor controlled PLC and PLC automatically depending on distance. Therefore, the key is to control the position, especially in the direction of walking. In the paper, the servo motor with absolute value 
encoder is adopted, through the match between the gear of the intelligent transfer device and the rack on running rail, so the position of the device can be quickly and accurately read out, and it is easy to carry out the position control and speed control.

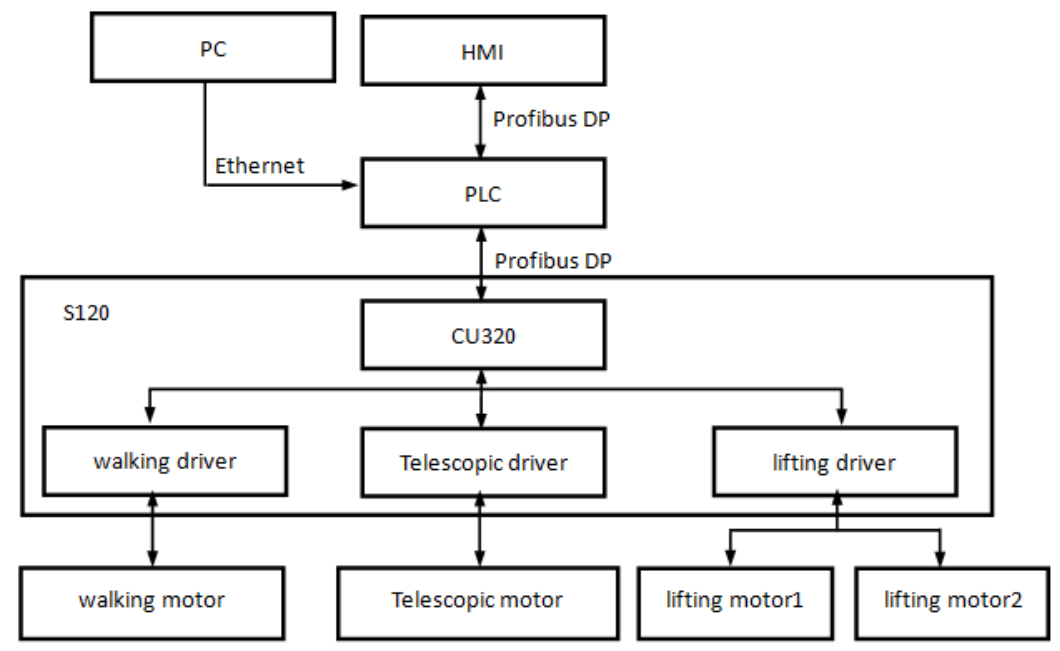

Fig.1 The structure of control system

In order to facilitate the description of the program, the field station layout is simplified as shown in Fig. 2.

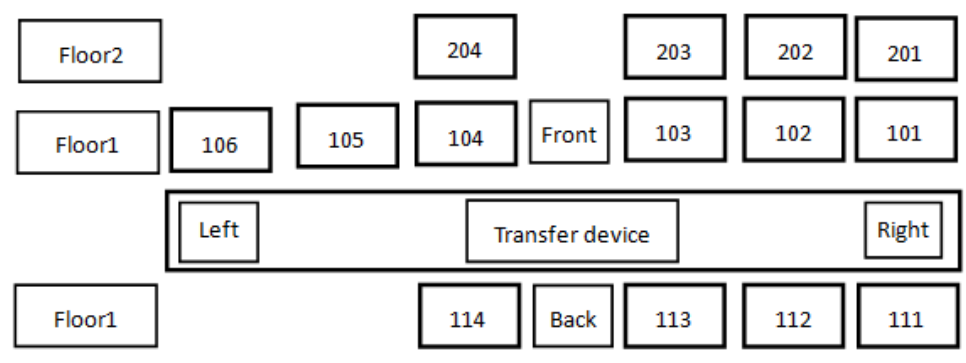

Fig.2 The simplified field station layout

It is assumed that the production line mainly includes two kinds of different products, which are A, B. The simplified logistics process is shown in Tab.1.

Tab.1 Simplified logistics process

\begin{tabular}{ccccccc}
\hline & \multicolumn{3}{c}{ Workpiece A } & & \multicolumn{3}{c}{ Workpiece B } \\
\cline { 3 - 6 } Take out & Logistics & Discharge & Take out & Logistics & Discharge \\
station & direction & station & station & direction & station \\
\hline 101 & $\rightarrow$ & 111 & 103 & $\rightarrow$ & 113 \\
111 & $\rightarrow$ & 102 & 113 & $\rightarrow$ & 104 \\
102 & $\rightarrow$ & 112 & 104 & $\rightarrow$ & 114 \\
112 & $\rightarrow$ & 201 & 114 & $\rightarrow$ & 203 \\
201 & $\rightarrow$ & 202 & 203 & $\rightarrow$ & 204 \\
202 & $\rightarrow$ & 105 & 204 & $\rightarrow$ & 106 \\
\hline
\end{tabular}

Under the condition of movement, speed, position control function realization, according to the requirement of the equipment, the equipment is set up to four modes, such as maintenance mode, manual mode, semi-automatic mode and automatic mode. Among them, the maintenance mode has the highest authority, only the system design engineers and debugging engineers can operate, its basic function is mainly to debug equipment failure and modify driving parameters. In the manual mode, each movement can be operated separately, and the main functions include speed change, 
position data modification, point move, etc. In the semi-automatic mode, the synthesis of partial actions can be completed. For example, taking and discharge movements are realized through the synthesis of $\mathrm{X}$ direction, $\mathrm{Y}$ direction, $\mathrm{Z}$ direction movements. Automatic mode does not need manual intervention, the device will automatically run. When the demand of a certain position is send out, the system will automatically determine the current state of the device. If the device is in idle state, the device performs the command, or the device executes the corresponding commands in accordance with the demands. In order to optimize program structure and realize the system control, the paper uses the modular structure design, each motion control function module is solidified and the system calls the relevant procedures according to needs. The main program diagram is shown in Fig.3.

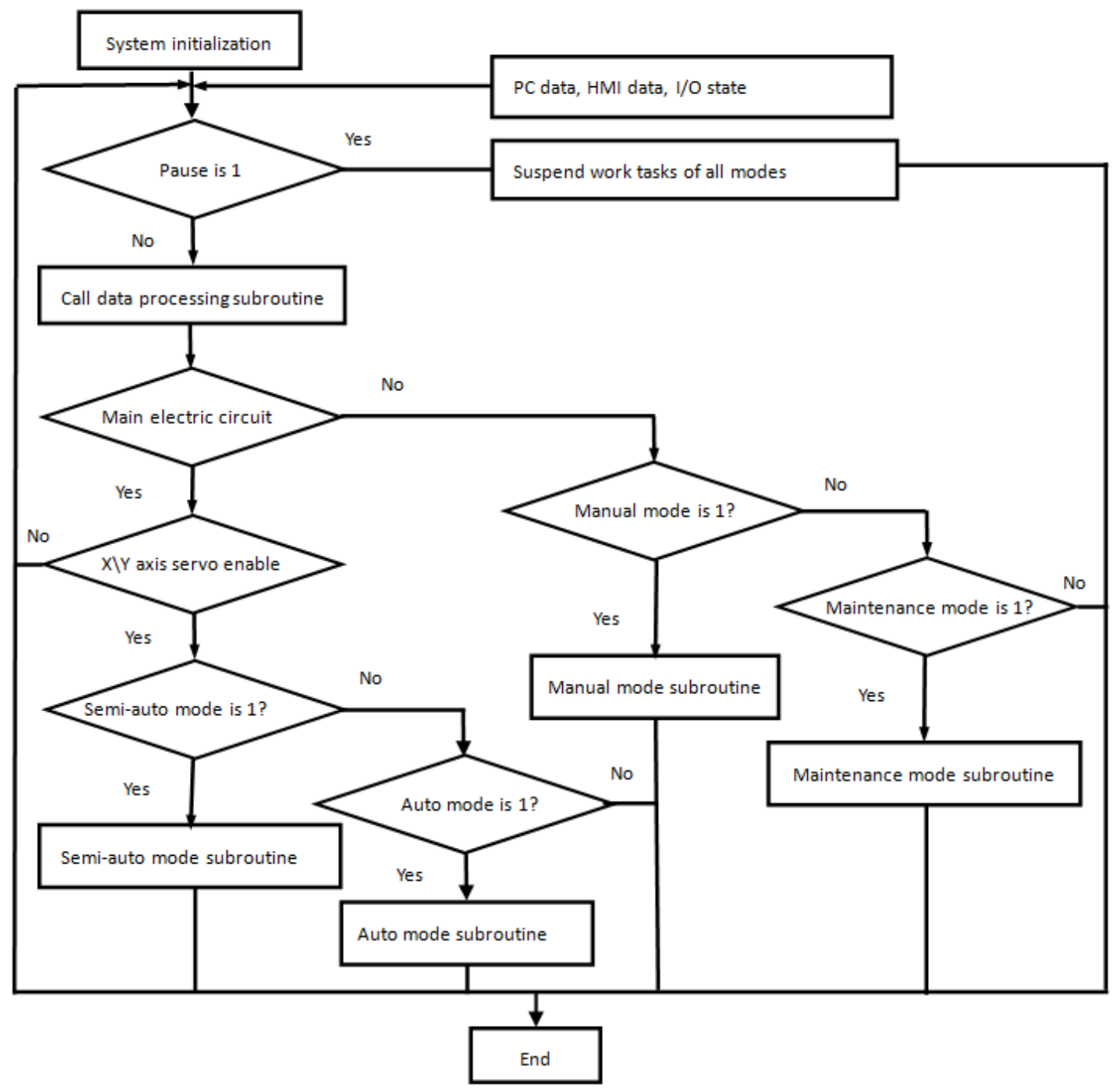

Fig.3 The main program diagram

The diagram of automatic mode program is shown in Fig.4. The mode mainly realizes the reservation of the station information, and executes the corresponding control action in accordance with the order. For example, there is a need to take the workpiece A from the 101 station to the 111 station. When the workpiece A is placed to the 101 station, the station will send out the demand to carry. At this point, the system will determine whether there is workpiece on the 111 station or not at once. If there is, the program will wait. If not, the system will drive the $\mathrm{X}$ axis to run to the 101 station, and then start taking subroutine. When taking workpiece is completed, then the $\mathrm{X}$ axis is driven to run to the 111 station, and then perform the discharge subroutine, so, it will complete the reservation information of the workpiece A from the station 101 to station 111 . Other modes are 
similar to this. In Figure 4, "[101] is 1" indicates that the 101 station has issued an appointment order and the 111 station has no workpiece, the rest is similar.

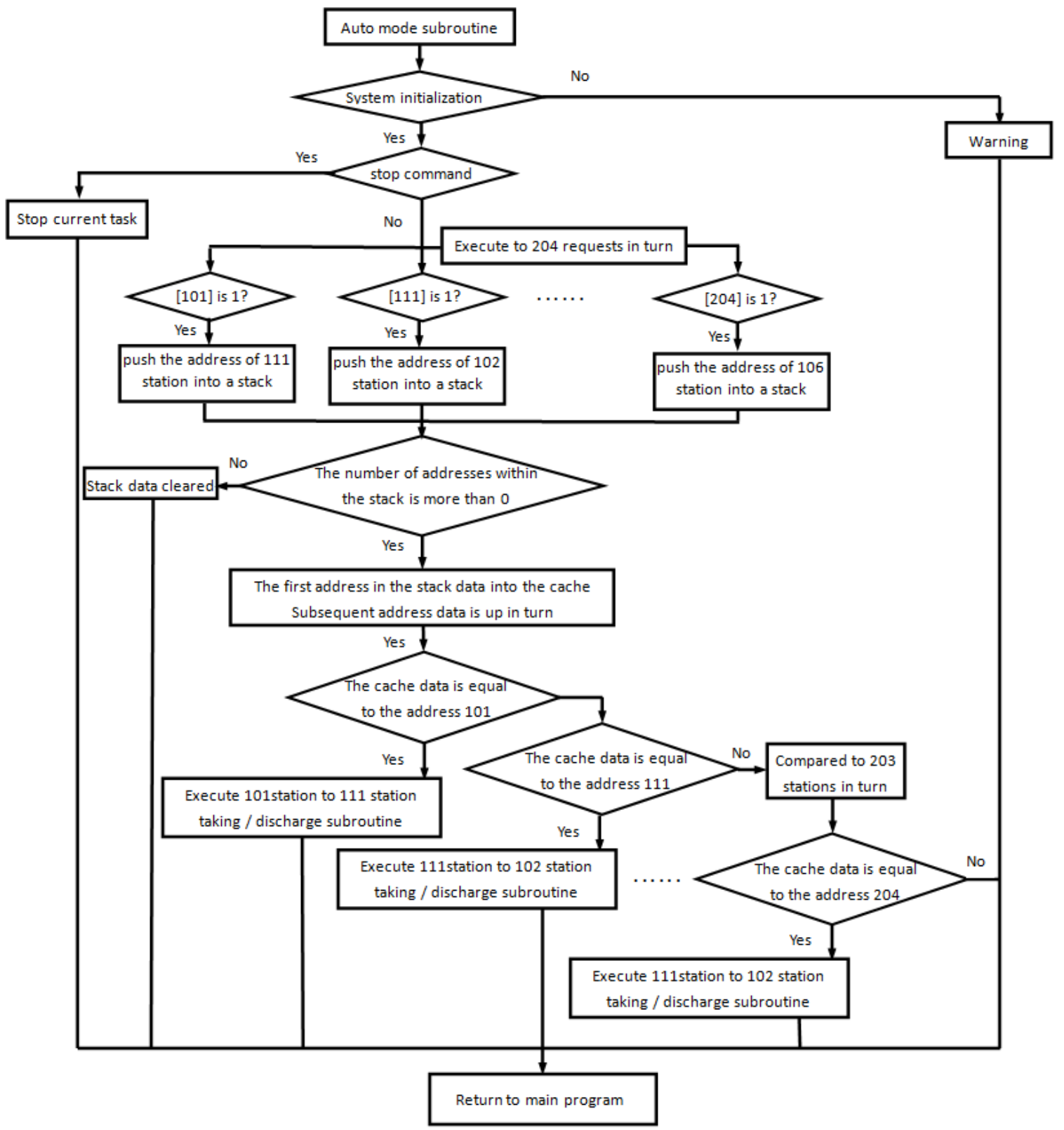

Fig.4 The automatic mode program diagram

According to the flow chart of main program and the process of their respective work mode, the paper uses the flexible WINCC interface software to design and develop the interfaces of four different mode conditions. Fig.5 shows the man-machine operation interface of the intelligent transfer device under automatic mode conditions. In Fig.5, the system is in the state of power, the X axis and the $\mathrm{Y}$ axis are in the state of the enable, and the device is in the state of rest in automatic mode. Through field debugging, the control function of the intelligent transfer device can be realized, which can satisfy the requirement of the intelligent transfer device. 


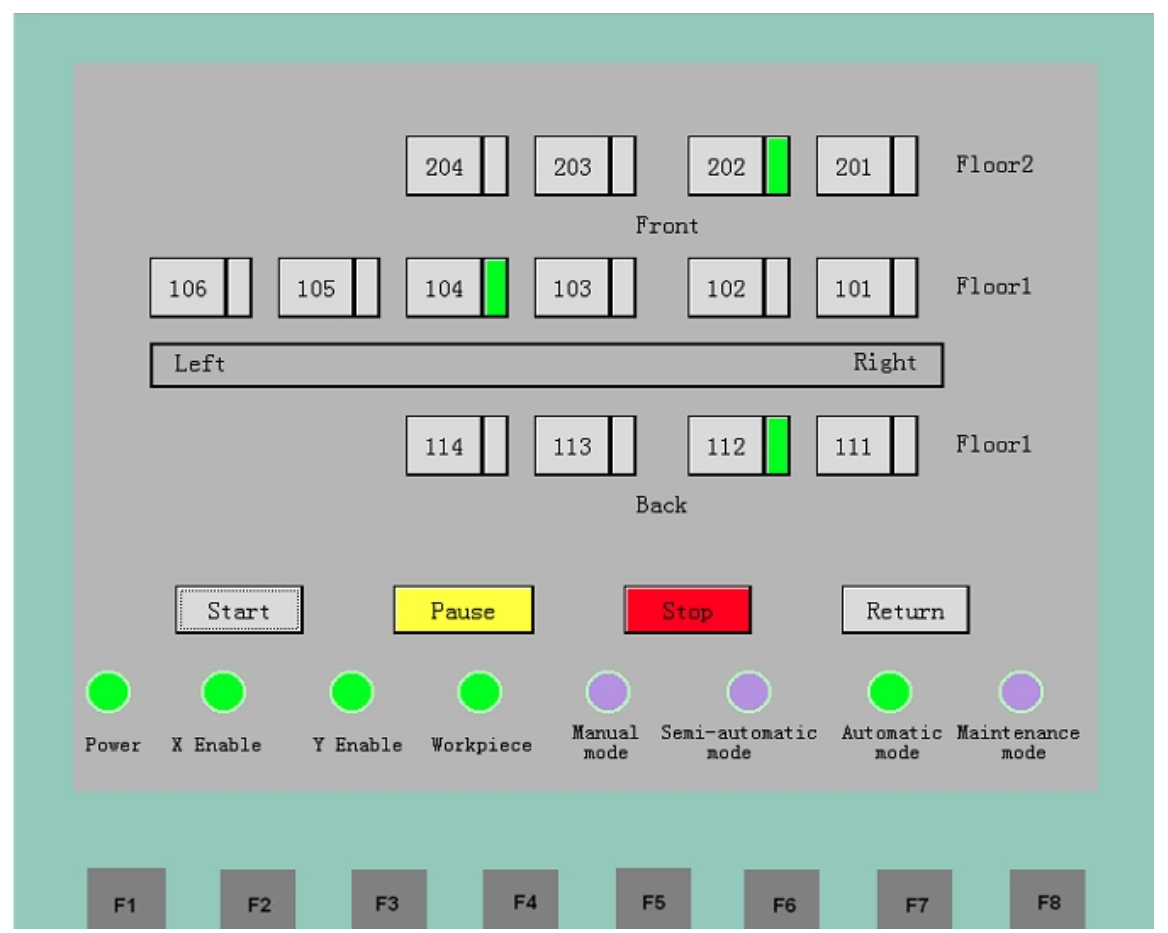

Fig.5 The automatic mode of human-machine interface

\section{conclusions}

A control system of the intelligent transfer device based on S120 and S7-300PLC is developed in the paper, which is equipped with the operation interfaces of touch screen and the host computer. After debugging and trial operation, the system can meet the requirements of the smart transfer device.

\section{Reference}

[1] ZHANG Chenbeixi, HUANG Zhi-qiu. Evolution Summarization of Automated Guided Vehicles (AGV)[J]. Manufacture Information Engineering of China, 2010, 39(1): 53-59.(In Chinese)

[2] Hu Gui Yan,Sun Wei Hua,Song Yu Qing. Study on Sustainable Development of China's Automated Stereoscopic Warehouse Building[J]. Advanced Materials Research . 2012 (524)

[3] E.H. Frazelle. World-Class Warehousing and Material Handling. 2001

[4] NIE Xin-nian. Roadway Stacker Control System Design [J]. Equipment Manufacturing Technology, 2010, (5): 64-66.(In Chinese)

[5]Zhou qi-cai. Study on Control and Management Techniques of Automated Storage and Retrieval System (AS/RS) Basing on Modern Logistics [D].Southwest Jiaotong University, 2002(In Chinese)

[6]Li Xiaoping. Study on speed control and positioning method of stacker based on BPS[J]. Hoisting and Conveying Machinery,2008, (12): 13-15. (In Chinese)

[7]Kodagoda S, Wijesorna S, Teoh K .Fuzzy speed and steering control of an AGV[J] .IEEE Transact ions on Control System Technology , 2002, 10(1):112 -120 